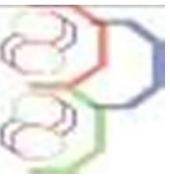

Journal of Applied Biosciences 149: 15297 - 15309

\title{
Etude diachronique de la forêt communautaire d'Ahouanhouzoukan à Tévèdji dans la Commune de Ouinhi au Sud-Bénin
}

Frida Kobayé OBOSSOU1, Jacques Boco ADJAKPA ${ }^{1}$, Jean Bosco Kpatindé VODOUNOU², Florent Essin DISSOU1, Mathias Fanou DANSI ${ }^{1}$, HounnankponYEDOMONHAN ${ }^{3}$

1 Université d'Abomey-Calavi (UAC), Ecole Polytechnique d'Abomey-Calavi (EPAC), Laboratoire de Recherche en Biologie Appliquée (LARBA), Bénin

2Université de Parakou, Faculté des Lettres, Arts et Sciences Humaines (FLASH), Département de Géographie et d'Aménagement du Territoire, Bénin

3Université d'Abomey-Calavi (UAC), Faculté des Sciences et Techniques (FAST), Laboratoire de Botanique et Ecologie Végétale (LABEV), Bénin

*Auteur correspondant : kofreeda@gmail.com

Original submitted in on $13^{\text {th }}$ February 2020. Published online at www.m.elewa.org/journals/ on $31^{\text {st }}$ May 2020

https://doi.org/10.35759/JABs.149.4

\section{RÉSUMÉ}

Contexte : Les forêts communautaires sont des réservoirs de biodiversité. L'étude a été conduite dans la forêt communautaire d'Ahouanhouzoukan située dans la Commune de Ouinhi au Sud-Bénin dont aucune étude n'a portée sur la cartographie des unités d'occupation du sol et la dynamique temporelle.

Objectif : Cette étude vise à évaluer la dynamique temporelle de cette forêt.

Méthodologie et Résultats : La méthode de collecte de données a combiné l'exploitation de trois images satellitaires multi spectrales Landsat de 1986, 2000 et 2013 et un contrôle terrain. Les résultats ont révélé que cette forêt présente quatre principales unités d'occupation du sol. II s'agit des forêts riveraines, des jachères, des forêts riveraines dégradées et des formations marécageuses. II ressort que de 1986 à 2013, les forêts riveraines et les jachères ont connu une diminution respective de leur superficie de 210,88 ha et 10,34 ha alors que les forêts riveraines dégradées et les formations marécageuses en ont connu une augmentation respective de 198,28 ha et 22,95 ha.

Conclusion : Cette étude fournit les premières données sur la cartographie de cette forêt et révèle une dégradation des forêts riveraines. II urge d'investiguer sur les causes de cette dégradation.

Mots clés : Diachronie, forêt communautaire, Ouinhi, Sud-Bénin

\section{ABSTRACT}

Context: Community forests are reservoirs of biodiversity. The study was conducted in Ahouanhouzoukan community forest located in Ouinhi a municipality of southern Benin in which there is no study regarding cartography of use land units and temporal dynamic.

Objective: It aims to evaluate spatial dynamics of this forest.

Méthodology and Results: The data collection method combined exploitation of three Landsat multi spectral satellite images of 1986, 2000 and 2013 and field monitoring. The results revealed that this forest has four main land use units. These include riparian forests, fallows, degraded riparian forests and wetlands. From 1986 to 2013, riparian forests and fallow land decreased respectively by 210.88 ha and 10.34 ha, while degraded riparian forests and marsh formations increased by 198.88 ha, 28 ha and 22.95 ha. 
Conclusion: This study provide the first cartographic data of this forest and show the degradation of riparian forests. It is necessary to investigate on the causes of this degradation.

Key words: Diachrony, community forest, Ouinhi, South Benin

\section{INTRODUCTION}

Les forêts constituent une composante majeure de l'environnement à cause de leur importance dans la protection des sols, des écosystèmes et de la faune (Arouna et al., 2011) et représentent un indice d'évaluation des conditions écologiques et des activités humaines (Orthmann, 2005). De ce fait, elles sont au cœur des enjeux internationaux sur la conservation de la biodiversité (de Wasseige et al., 2013). Cependant, elles connaissent aujourd'hui sous l'effet combiné des changements climatiques et des activités humaines une régression spectaculaire de leur superficie (Noumi et al., 2011). En Afrique en général et au Bénin en particulier, les causes de cette perte de superficie sont les mêmes que celles relevées dans le reste du monde avec le développement de l'agriculture (FAO, 2016). Selon Geist \& Lambin (2001), plusieurs causes directes telles que l'agriculture itinérante, la collecte du bois énergie, l'exploitation minière, l'exploitation forestière et le développement des infrastructures expliquent la

\section{MATERIEL ET METHODES}

Milieu d'étude : L'étude a été conduite dans la forêt communautaire d'Ahouanhouzoukan localisée entre $7^{\circ} 035$ minutes et $7^{\circ} 058$ minutes de latitude Nord et $2^{\circ} 22$ minutes et $2^{\circ} 25$ minutes de longitude Est et située à l'Ouest de la Commune de Ouinhi, précisément à Tévedji dans l'Arrondissement de Sagon (figure 1). Le relief est constitué essentiellement de bas plateaux. Les sols sont hydromorphes et halomorphes établis sur matériau alluvial, argileux et sablo-argileux (Adjakpa, 2006). Le climat est du type subéquatorial avec une pluviosité moyenne annuelle dégradation forestière. Selon ces auteurs, il existe aussi des causes structurelles ou sous-jacentes comme la croissance démographique, les facteurs macroéconomiques (le produit intérieur brut, la production industrielle, le taux de chômage), la déstructuration des sociétés traditionnelles et la mutation des pratiques traditionnelles. Cependant, la pression humaine due à la poussée démographique est d'autant plus importante que certaines espèces et même des écosystèmes sont actuellement en voie de disparition (Tenté, 2005). La forêt communautaire d'Ahouan houzoukan dans la Commune de Ouinhi est une relique de forêt peu connue qui suscite l'admiration et la curiosité des populations riveraines. Elle a fait l'objet de très peu d'études or l'élaboration d'un plan d'aménagement de ladite forêt doit passer par la disposition de données relatives à sa superficie, les différentes formations végétales qu'elle présente et sa dynamique. C'est dans ce contexte que s'inscrit la présente étude.

de $1250 \mathrm{~mm}$ avec deux saisons sèches et deux saisons pluvieuses. Les températures annuelles moyennes varient entre $29,8{ }^{\circ} \mathrm{C}$ et $36,3{ }^{\circ} \mathrm{C}$ sans grande variation diurne ou saisonnière. La végétation est constituée de formations marécageuses, de forêts galeries, de prairies boisées et de forêts galeries. La faune est constituée de mammifères, d'oiseaux, de reptiles, d'insectes et de poissons. La population de la Commune de Ouinhi est estimée à 59381 habitants et celle du village de Tévèdji est de 1755 habitants et elles sont essentiellement agricoles (INSAE, 2013). 


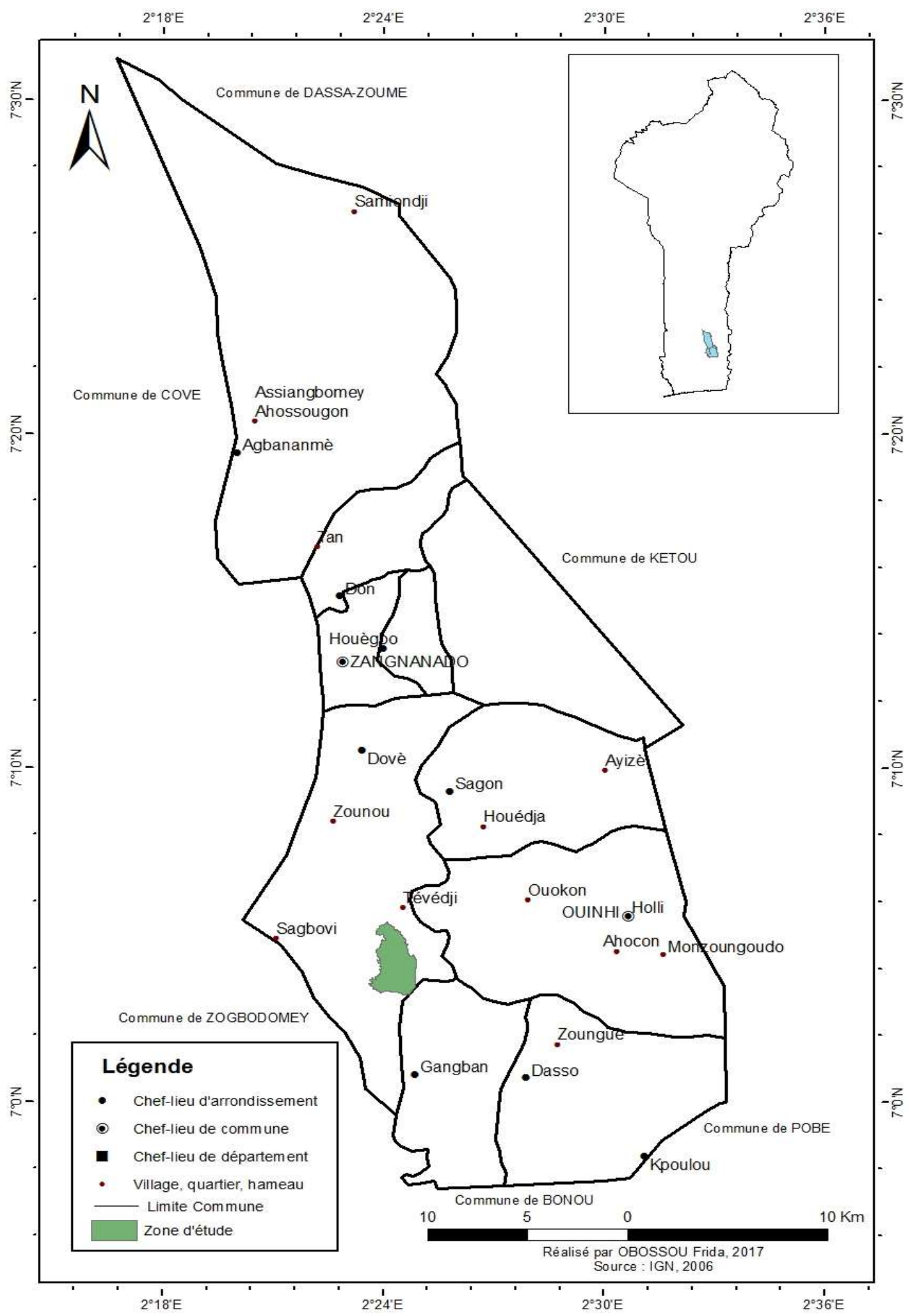

Figure 1. Localisation géographique de la forêt d'Ahouanhouzoukan dans la Commune de Ouinhi 
Matériel : Deux types de matériels ont été utilisés : un matériel biologique et un matériel technique. Le matériel biologique est constitué de la forêt communautaire d'Ahouanhouzoukan. Le matériel technique est constitué d'un GPS (Global Positioning System) et d'un appareil photo numérique. Une carte topographique au $1 / 200000$, feuille ABOMEY NB -31 XX-XX I République du Bénin et trois images satellitaires Landsat: LT51920551986317XXX03 du 13/11/1986, LE71920552000341EDC00 du 13/ 12/2000 et LC81920552013343LGN00 du 09/12/2013 ont également été utilisées.

Méthode

Collecte des données : Les données proviennent de L 1-5 MSS (Multi Spectral Scanner) pour l'image de 1986, L7 ETM+ SCL-on (1999-2003) [Enhanced Thematic Mapper Plus Scan Line Corrector] pour l'image de 2000 et L8 OLI/TIRS (Operational Land Imager/Thermal InfraRed Sensor) pour l'image de 2013. L'image de 1986 a une résolution de $57 \times 79 \mathrm{~m}$ ; celle de 2000, $30 \mathrm{~m}$ pour le réflectif et $60 \mathrm{~m}$ pour le thermique (Gyanesh et al., 2009) et enfin celle de $2013,30 \mathrm{~m}$ pour le réflectif et $100 \mathrm{~m}$ pour le thermique. Une mission de vérification et d'étude de terrain a également été effectuée. Au cours de cette dernière, un tracking a été fait autour de la forêt pour évaluer sa superficie et relever les coordonnées des unités d'occupation du sol. La nomenclature utilisée pour l'identification des formations végétales est celle de Yangambi (Aubreville, 1957).

Traitement et analyse des données : La méthode est centrée sur le traitement et l'interprétation d'images. Elle a consisté à l'importation et la combinaison des bandes à l'aide du logiciel MultiSpec afin de constituer une image multi spectrale. Ensuite, la zone d'intérêt a été extraite à partir de la fonction Reformat de

\section{RESULTATS ET DISCUSSION}

\section{Résultats}

Analyse de la précision thématique des classifications: Pour chacune des images, la précision de la classification supervisée a été analysée afin de rechercher si les unités d'occupation sont bien rangées et par conséquent si l'espace de la forêt est
MultiSpec pour faciliter le processus de traitement. Une classification supervisée des images Landsat 1986, 2000 et 2013 a été effectuée sur la base des informations collectées sur le terrain. Ainsi, l'indice Kappa évalue dans la matrice de confusion l'accord entre les résultats obtenus (carte) et la vérité sur le terrain (Bogaert et al., 2011). L'indice Kappa (équation 1) est exprimé en termes de précision globale observée (équation 2) et de précision prévue (équation 3).

$$
\begin{aligned}
& \widehat{\mathrm{K}}=\frac{\mathrm{a}-\mathrm{b}}{1-\mathrm{b}} \widehat{\mathrm{K}}=\frac{\mathrm{a}-\mathrm{b}}{1-\mathrm{b}} \\
& \mathrm{a}=\frac{1}{\mathrm{~N}} \sum_{\mathrm{i}=1}^{\mathrm{Nc}} \mathrm{Xiia}=\frac{1}{\mathrm{~N}} \sum_{\mathrm{i}=1}^{\mathrm{N} z} \mathrm{Xiil}_{\text {ii }} \text { (2) } \\
& \mathrm{b}=\frac{1}{\mathrm{~N}^{2}} \sum_{\mathrm{i}=1}^{\mathrm{Nc}}(\mathrm{X}+\mathrm{i} \cdot \mathrm{Xi}+) \\
& \mathrm{b}=\frac{1}{\mathrm{~N}^{2}} \sum_{\mathrm{i}=1}^{\mathrm{Nc}}(\mathrm{X}+\mathbf{i} . \mathrm{Xi}+)
\end{aligned}
$$

$N c=$ Nombre de classes; $N=$ Nombre total d'observations ; ii $X=$ Nombre d'observations dans la colonne i ligne $i$ (diagonale de la matrice) ; $i X_{+}=$Total des observations dans la colonne $i$ (total à droite dans la matrice) ; $i+X=$ Total des observations dans la ligne $i$ (total en bas de la matrice).

Cet indice varie de 0 à 1 et il se subdivise en cinq catégories : accord très faible de 0 à 0,20 ; accord faible de 0,21 à 0,40 ; accord modéré de 0,41 à 0,60 ; accord substantiel de 0,61 à 0,80 ; accord presque parfait de 0,81 à 1 .

Enfin, les images rasters issues de la classification sur MultiSpec, ont été importées dans ArcMap de ArcGIS pour la représentation cartographique. Ces images rasters ont été converties en fichiers vectoriels afin de déterminer les superficies des différentes unités d'occupation du sol.

bien représenté. Les tableaux 1, 2 et 3 montrent pour chacune des classes, le niveau de fiabilité moyen et les principales confusions observées.

Image MSS-1986 : La matrice de confusion de l'image MSS-1986 est présentée par le Tableau 1.

Tableau 1 : Matrice de confusion de l'image MSS-1986

\begin{tabular}{|l|l|l|l|l|l|l|l|}
\hline Classes & FR à C. laurifolia & FR à M. inermis & J & FM & CE & Total & Précision \\
\hline FR à C. laurifolia & 1637 & 2 & 1 & 0 & 0 & 1640 & 99,8 \\
\hline FR à M. inermis & 0 & 34 & 0 & 0 & 0 & 34 & 100 \\
\hline J & 0 & 0 & 432 & 0 & 0 & 432 & 100 \\
\hline FM & 0 & 0 & 0 & 9 & 0 & 9 & 100 \\
\hline CE & 0 & 0 & 0 & 0 & 12 & 12 & 100 \\
\hline
\end{tabular}

FR : Forêt Riveraine à Cola laurifolia ; FR : Forêt Riveraine à Mitragina inermis ; J : Jachère ; FM : Forêt Marécageuse ; CE : Cours d'Eau. 
La matrice de confusion de l'image MSS-1986 donne une précision globale de la cartographie de 99,9\%. La précision absolue (100\%) est observée au niveau des classes FR à $M$. inermis, J, FM et CE. Ensuite vient la classe FR à C. laurifolia $(99,8 \%$ ) qui s'est entremêlée de façon négligeable. En effet, la classification obtenue est significativement réel (indice Kappa $=99,6 \%$ ). II existe donc un accord substantiel entre les résultats obtenus par classification (figure 2 ) et les réalités du terrain (Kappa Variance $=0,000005$ ).

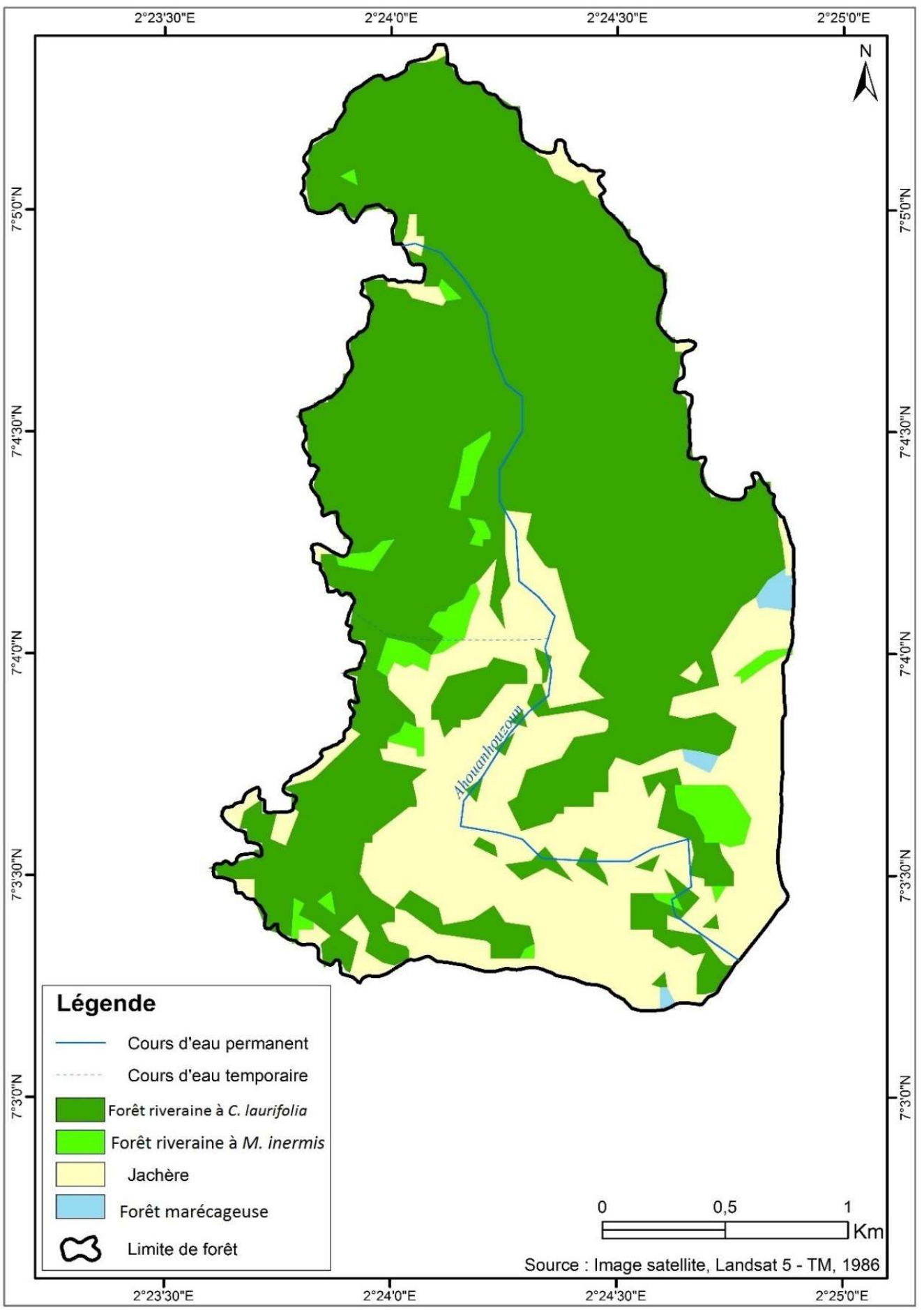

Figure 2. Classification supervisée de l'image MSS-1986

Cette classification permet d'avoir une idée sur l'occupation du sol du secteur d'étude en 1986. Cette occupation du sol se voit dominée par la forêt riveraine à $C$. laurifolia illustrée en vert foncé.

Image ETM+SCL-2000 : Le Tableau 2 suivant présente la matrice de confusion de l'image ETM+SCL-2000. 
Obossou et al., J. Appl. Biosci. 2020 Etude diachronique de la forêt communautaire d'Ahouanhouzoukan à Tévèdji dans la Commune de Ouinhi au Sud-Bénin

Tableau 2 : Matrice de confusion de l'image ETM+SCL-2000

\begin{tabular}{|l|l|l|l|l|l|l|l|}
\hline Classes & FR à C. laurifolia & FR à M. inermis & J & FM & CE & Total & Précision \\
\hline FR à C. laurifolia & 857 & 1 & 0 & 0 & 0 & 858 & 99,9 \\
\hline FR à M. inermis & 0 & 30 & 2 & 0 & 0 & 32 & 93,8 \\
\hline J & 0 & 0 & 246 & 0 & 0 & 246 & 100 \\
\hline FM & 0 & 0 & 2 & 153 & 0 & 155 & 98,7 \\
\hline CE & 0 & 0 & 0 & 0 & 28 & 28 & 100 \\
\hline
\end{tabular}

FR : Forêt Riveraine à Cola laurifolia ; FR : Forêt Riveraine à Mitragina inermis ; J : Jachère ; FM : Forêt Marécageuse ; CE : Cours d'Eau.

La matrice de confusion de l'image ETM+SCL-2000 indique une précision globale de la cartographie de $99,6 \%$. Dans le détail, on observe que les classes J et $C E$ influencent fortement l'évaluation globale de la précision car elles obtiennent une précision élevée $(100 \%)$. Viennent ensuite les classes FR à C. laurifolia $(99,9 \%)$, FM $(98,7 \%)$, FR à M. inermis $(93,8 \%)$ qui sont respectivement confondues par les échantillons de la FR à $M$. inermis de $0,01 \%$, des $\mathrm{J}$ de $1,3 \%$ et 6,2 $\%$. La classification effectuée est correcte à 99,3\% (indice kappa). II existe alors un accord substantiel entre les résultats obtenus par classification (Figure 3) et la vérité sur le terrain ce que montre bien la variance $(0,000010)$. 


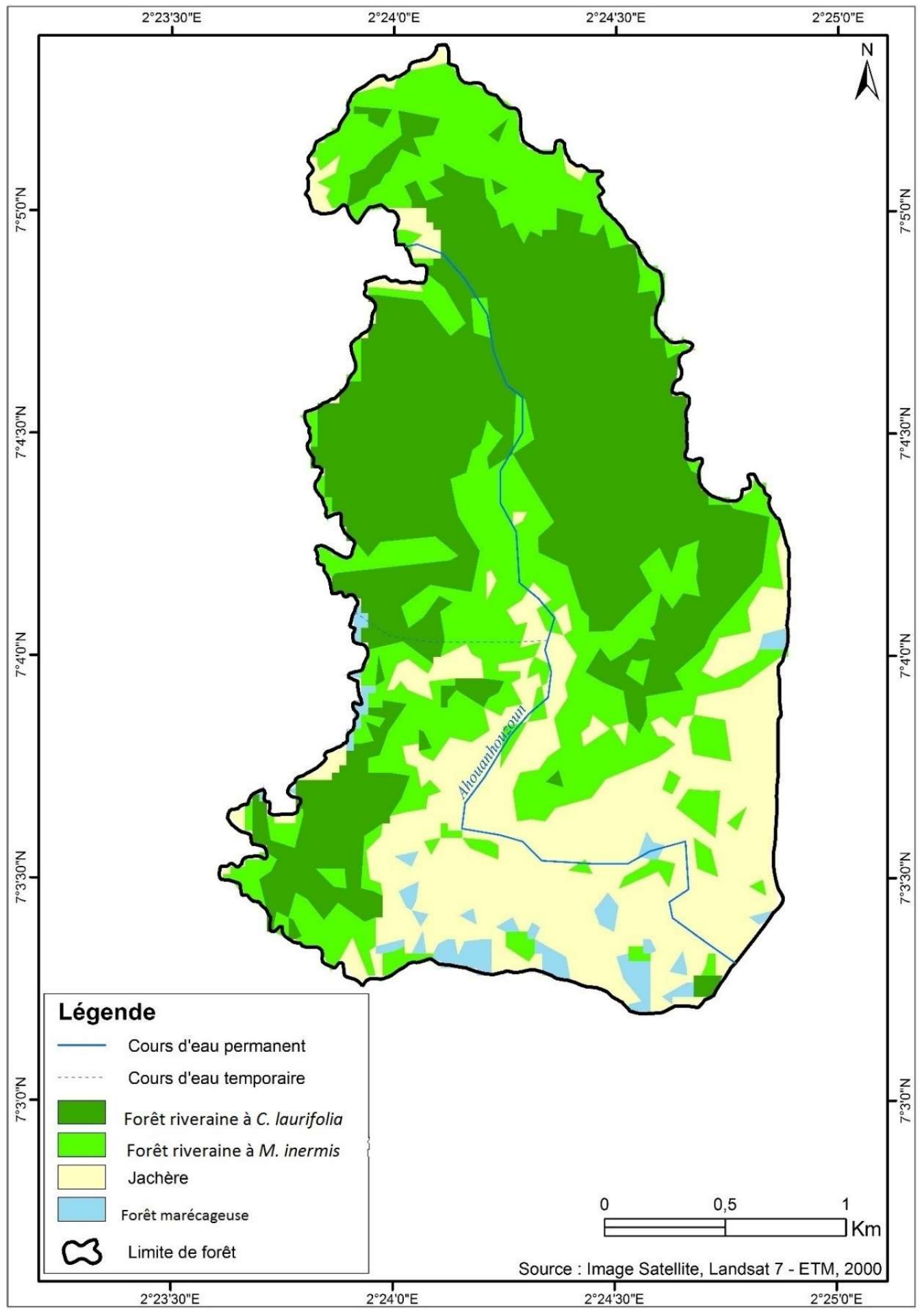

Figure 3. Classification supervisée de l'image ETM+SCL-2000

Cette classification permet d'avoir une idée sur l'occupation du sol du secteur d'étude en 2000. Cette occupation du sol se voit aussi dominée par la forêt riveraine à $C$. laurifolia illustrée en vert foncé.

Image OLI/TIRS-2013 : Le Tableau 3présente la matrice de confusion de l'image OLI/TIRS-2013. 
Obossou et al., J. Appl. Biosci. 2020 Etude diachronique de la forêt communautaire d'Ahouanhouzoukan à Tévèdji dans la Commune de Ouinhi au Sud-Bénin

Tableau 3 : Matrice de confusion de l'image OLI/TIRS-2013

\begin{tabular}{|l|l|l|l|l|l|l|l|}
\hline Classes & FR à C. laurifolia & FR à M. inermis & J & CE & FM & Total & Précision \\
\hline FR à C. laurifolia & 450 & 1 & 0 & 0 & 0 & 451 & 99,8 \\
\hline FR à M. inermis & 0 & 71 & 1 & 0 & 0 & 72 & 98,6 \\
\hline J & 0 & 3 & 193 & 0 & 0 & 196 & 98,5 \\
\hline CE & 0 & 0 & 0 & 15 & 0 & 15 & 100 \\
\hline FM & 0 & 0 & 1 & 0 & 31 & 32 & 96,9 \\
\hline
\end{tabular}

FR: Forêt Riveraine à Cola laurifolia ; FR: Forêt Riveraine à Mitragina inermis ; J : Jachère ; FM : Forêt Marécageuse ; CE : Cours d'Eau.

La matrice de confusion de l'image OLI/TIRS-2013 indique une précision globale de la cartographie de $99,2 \%$. Dans le détail, on observe que la classe CE influence grandement l'évaluation globale de la précision car elle obtient une précision élevée (100\%). Viennent ensuite les classes FR à C. laurifolia $(99,8$ $\%)$, FR à M. inermis (98,6\%), J (98,5\%) et FM (96,9
\%) qui sont aussi moins affectés par les échantillons des autres classes. La classification effectuée est correcte à 98,6\% (indice kappa). II existe alors un accord substantiel entre les résultats obtenus par classification (Figure 4 ) et la vérité sur le terrain ce que montre bien la variance $(0,000030)$. 


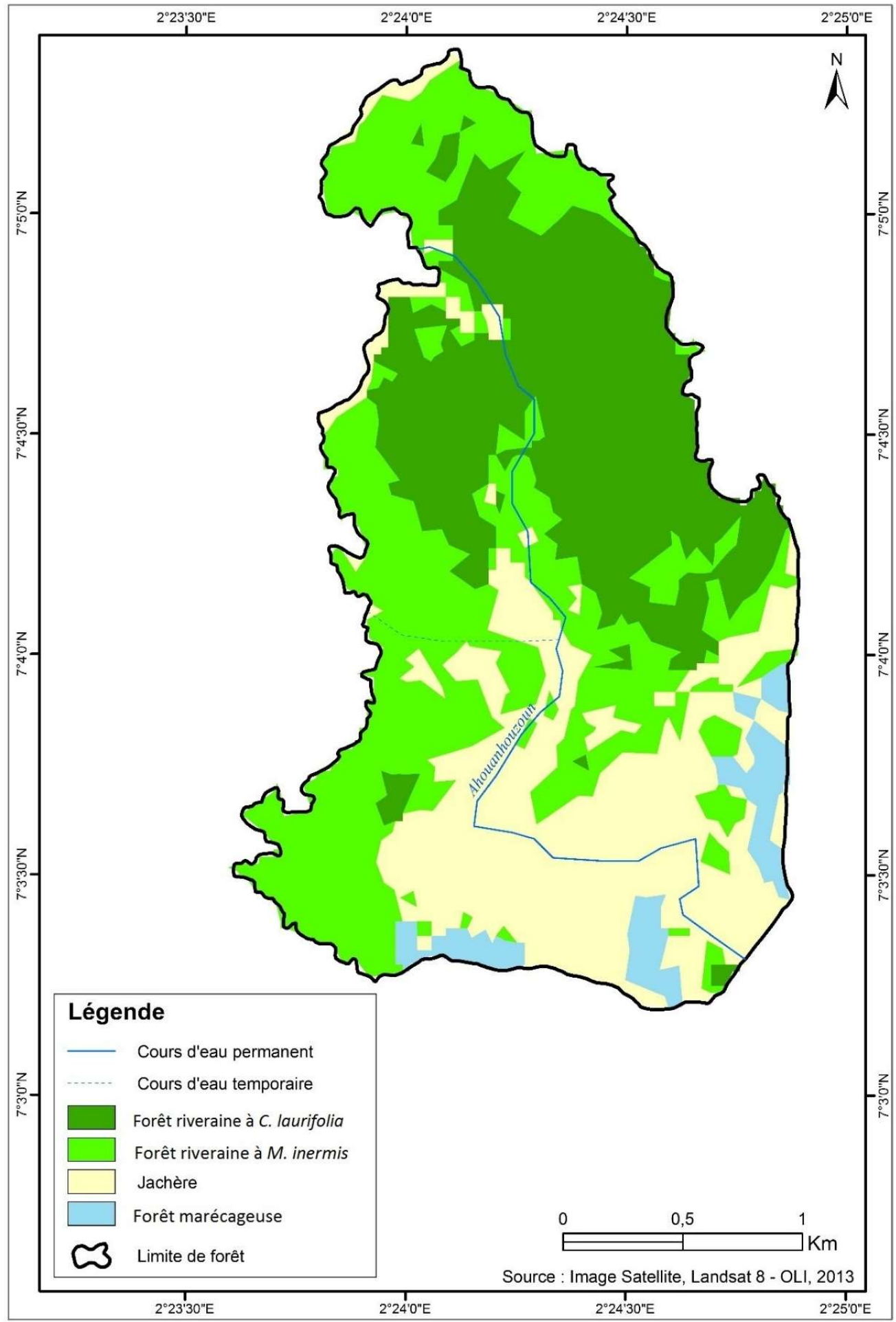

Figure 4. Classification supervisée de l'image OLI/TIRS-2013

Cette classification permet d'avoir une idée sur l'occupation du sol du secteur d'étude en 2013. Les forêts riveraines à C. laurifolia ont diminuées de superficie au détriment des forêts riveraines à $M$. inermis illustrée en vert claire.

Etats de l'occupation du sol

Occupation du sol en 1986: En 1986, la zone d'étude présentait les unités d'occupation du sol suivantes (figure 2):
- les forêts riveraines à C. laurifolia couvraient 393,99 ha soit $66,36 \%$ de la superficie totale de la forêt ; 
- les forêts riveraines à $M$. inermis représentaient 19,15 ha soit $3,23 \%$ de la superficie totale de la forêt ;

- les jachères occupaient une surface de 177,43 ha soit $29,88 \%$ de la superficie totale de la forêt ;

- enfin les forêts marécageuses totalisaient 3,14 ha correspondant à un pourcentage de $48,34 \%$ de la forêt.

Occupation du sol en 2000 : Les calculs effectués sur la carte d'occupation du sol de 2000 (figure 3) ont permis de déterminer la superficie de chaque classe. Au cours de cette année :

- les forêts riveraines à $C$. laurifolia couvraient une aire de 241,97 ha représentant 40,76\% de la superficie totale de la forêt ;

- les forêts riveraines à $M$. inermis correspondaient à une superficie de 172,58 ha soit $29,07 \%$ de la surface totale de la forêt ;

- les jachères représentaient 164,38 ha soit $27,69 \%$ de la surface totale ;

- les forêts marécageuses totalisaient une superficie de 14,77 ha soit $2,49 \%$ de la superficie totale de la forêt.

Occupation du sol en 2013 : La carte d'occupation du sol de 2013 (figure 4) révèle une occupation en 5 classes réparties de la façon suivante :

- les forêts riveraines à $C$. laurifolia occupaient une superficie de 183,10 ha, ce qui représente $30,84 \%$ de la superficie totale de la forêt ;

- les forêts riveraines à $M$. inermis représentaient une superficie de 217,43 ha équivalant à $36,62 \%$ de la forêt ;

- les forêts marécageuses couvraient 26,09 ha soit un taux de $4,39 \%$ de la surface totale de la forêt ;

- enfin les jachères correspondaient à une superficie de 167,08 soit $28,14 \%$ de la forêt.

Dynamique de l'occupation du sol

De 1986 à 2000 : De 1986 à 2000 les différentes unités d'occupation du sol ont connu des évolutions spatiales. Les forêts riveraines à $C$. laurifolia ont connu une baisse de leur superficie de 1986 à 2000. Elles sont passées de 393,99 ha à 241,97 ha soit une réduction de 152,01 ha correspondant à une régression de 25,60 $\%$. Les jachères ont également perdu une partie de leur superficie; elles sont passées de 177,43 ha en 1986 à 164,38 ha en 2000 soit un taux de régression de $2,20 \%$. Contrairement aux forêts riveraines à $C$. laurifolia et aux jachères, les forêts marécageuses ont connues une augmentation. En 1986, la superficie qui était de 3,14 ha a atteint 14,77 ha en 2000 , ce qui correspond en valeur absolue en une augmentation de 11,63 ha soit un taux de 1,96\%. L'évolution la plus spectaculaire est réalisée par les forêts riveraines à $M$. inermis avec une superficie de 19,15 ha en 1986 pour atteindre 172,58 ha en 2000 , soit une augmentation de $25,84 \%$ (tableau 4).

Tableau 4 : Bilan des superficies et proportions des unités d'occupation du sol entre 1986 et 2000

\begin{tabular}{|c|c|c|c|c|c|c|}
\hline \multirow[b]{2}{*}{ Unités d'occupation } & \multicolumn{2}{|l|}{1986} & \multicolumn{2}{|l|}{2000} & \multicolumn{2}{|l|}{ Bilan } \\
\hline & $\begin{array}{l}\text { Superficie } \\
\text { (ha) }\end{array}$ & $\begin{array}{l}\text { Proportion } \\
(\%)\end{array}$ & $\begin{array}{l}\text { Superficie } \\
\text { (ha) }\end{array}$ & $\begin{array}{l}\text { Proportion } \\
(\%)\end{array}$ & $\begin{array}{l}\text { Superficie } \\
\text { (ha) }\end{array}$ & $\begin{array}{l}\text { Proportion } \\
(\%)\end{array}$ \\
\hline $\begin{array}{l}\text { Forêts riveraines à } C \text {. } \\
\text { laurifolia }\end{array}$ & 393,99 & 66,36 & 241,97 & 40,76 & $-152,01$ & $-25,60$ \\
\hline $\begin{array}{l}\text { Forêts riveraines à } M \text {. } \\
\text { inermis }\end{array}$ & 19,15 & 3,23 & 172,58 & 29,07 & 153,43 & 25,84 \\
\hline Jachères & 177,43 & 29,88 & 164,38 & 27,69 & $-13,05$ & $-2,20$ \\
\hline Forêts marécageuses & 3,14 & 0,53 & 14,77 & 2,49 & 11,63 & 1,96 \\
\hline Total & 593,70 & 100,00 & 593,70 & 100,00 & 0,00 & 0,00 \\
\hline
\end{tabular}

De 2000 à 2013 : De 2000 à 2013, les forêts riveraines à $C$. laurifolia ont perdu 58,87 ha de leur superficie soit un taux de régression de $9,92 \%$. Par contre, les forêts riveraines à $M$. inermis ont connu une augmentation, elles sont passées de 172,58 ha en 2000 à 217,43 ha en 2013 , soit une croissance de $7,55 \%$. Les forêts marécageuses et les jachères ont connu une tendance à la hausse de 2000 à 2013. Elles sont passées respectivement de 14,77 ha à 26,09 ha et de 164,38 ha à 167,08 ha soit une augmentation respective de 11,32 ha et 2,71 ha correspondant à un taux de 1,91 $\%$ et $0,46 \%$ (tableau 5 ). 
Tableau 5 : Bilan des superficies et proportions des unités d'occupation du sol entre 2000 et 2013

\begin{tabular}{|c|c|c|c|c|c|c|}
\hline \multirow[b]{2}{*}{ Unités d'occupation } & \multicolumn{2}{|l|}{2000} & \multicolumn{2}{|l|}{2013} & \multicolumn{2}{|l|}{ Bilan } \\
\hline & $\begin{array}{l}\text { Superficie } \\
\text { (ha) }\end{array}$ & $\begin{array}{l}\text { Proportion } \\
(\%)\end{array}$ & $\begin{array}{l}\text { Superficie } \\
\text { (ha) }\end{array}$ & $\begin{array}{l}\text { Proportion } \\
(\%)\end{array}$ & $\begin{array}{l}\text { Superficie } \\
\text { (ha) }\end{array}$ & $\begin{array}{l}\text { Proportion } \\
(\%)\end{array}$ \\
\hline $\begin{array}{l}\text { Forêts riveraines à } C \text {. } \\
\text { laurifolia }\end{array}$ & 241,97 & 40,76 & 183,10 & 30,84 & $-58,87$ & $-9,92$ \\
\hline $\begin{array}{l}\text { Forêts riveraines à } M \text {. } \\
\text { inermis }\end{array}$ & 172,58 & 29,07 & 217,43 & 36,62 & 44,85 & 7,55 \\
\hline Jachères & 164,38 & 27,69 & 167,08 & 28,14 & 2,71 & 0,46 \\
\hline Forêts marécageuses & 14,77 & 2,49 & 26,09 & 4,39 & 11,32 & 1,91 \\
\hline Total & 593,70 & 100,00 & \begin{tabular}{|l|}
593,70 \\
\end{tabular} & 100,00 & 0,00 & 0,00 \\
\hline
\end{tabular}

De 1986 à 2013 : A l'instar des deux premières périodes, les différentes unités d'occupation du sol ont connu des variations de leur superficie de 1986 à 2013. Les forêts riveraines à $C$. laurifolia ont vu leur superficie diminuer de 210,88 ha soit un taux de régression de $35,52 \%$. Pour ces formations, la phase critique de décroissance est celle de 1986 à 2000 au cours de laquelle elles ont perdu $25,60 \%$ de leur superficie. Les jachères sont passées de 177,43 ha à 167,08 ha soit une réduction de 10,34 ha correspondant à un taux de régression de $1,74 \%$. Contrairement, les forêts riveraines à $M$. inermis ont connu une augmentation de $33,40 \%$ par rapport à la superficie initiale en 1986. En effet, avec une superficie de 19,15 ha en 1986, elles atteignent 217,43 ha en 2013, ce qui correspond à une augmentation de 198,28 ha. Les forêts marécageuses ont aussi vu leur superficie croître. Elles sont passées de 3,14 ha en 1986 à 26,09 ha en 2013 , soit une augmentation de $3,87 \%$ (tableau 6).

Tableau 6. Bilan des superficies et proportions des unités d'occupation du sol entre 1986 et 2013

\begin{tabular}{|c|c|c|c|c|c|c|}
\hline \multirow[b]{2}{*}{ Unités d'occupation } & \multicolumn{2}{|l|}{1986} & \multicolumn{2}{|l|}{2013} & \multicolumn{2}{|l|}{ Bilan } \\
\hline & $\begin{array}{l}\text { Superficie } \\
\text { (ha) }\end{array}$ & $\begin{array}{l}\text { Proportion } \\
(\%)\end{array}$ & $\begin{array}{l}\text { Superficie } \\
\text { (ha) }\end{array}$ & $\begin{array}{l}\text { Proportion } \\
(\%)\end{array}$ & $\begin{array}{l}\text { Superficie } \\
\text { (ha) }\end{array}$ & $\begin{array}{l}\text { Proportion } \\
(\%)\end{array}$ \\
\hline $\begin{array}{l}\text { Forêts riveraines à } C \text {. } \\
\text { laurifolia }\end{array}$ & 393,99 & 66,36 & 183,10 & 30,84 & $-210,88$ & $-35,52$ \\
\hline $\begin{array}{l}\text { Forêts riveraines à } M \text {. } \\
\text { inermis }\end{array}$ & 19,15 & 3,23 & 217,43 & 36,62 & 198,28 & 33,40 \\
\hline Jachères & 177,43 & 29,88 & 167,08 & 28,14 & $-10,34$ & $-1,74$ \\
\hline Forêts marécageuses & 3,14 & 0,53 & 26,09 & 4,39 & 22,95 & 3,87 \\
\hline Total & 593,70 & 100,00 & 593,70 & 100,00 & 0,00 & 0,00 \\
\hline
\end{tabular}

Bilan de l'évolution des différentes unités d'occupation du sol de 1986, 2000 et 2013 : Les forêts riveraines à $C$. laurifolia et les jachères ont connu un recul très important de 1986 en 2013. Par contre, les forêts riveraines à $M$. inermis et les forêts marécageuses se sont étendues au détriment des forêts riveraines à $C$. laurifolia et les jachères comme l'indique la figure 5 . 


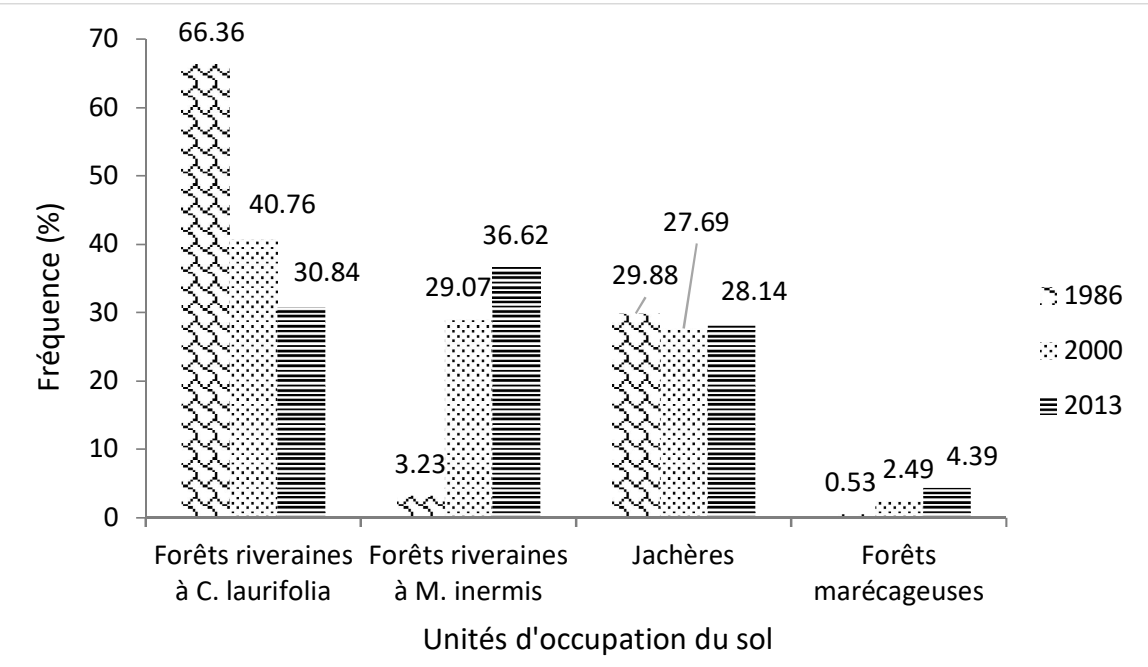

Figure 5 : Bilan de l'évolution des différentes unités d'occupation du sol de 1986 à 2013

\section{DISCUSSION}

Evolution de l'occupation des sols: L'analyse diachronique a montré que la forêt a connu une grande variation de la superficie de ces unités d'occupation du sol. De 1986 à 2013, les forêts riveraines à C. laurifolia ont connu une régression de $35,52 \%$ de leur superficie. Ceci peut s'expliquer par l'exploitation abusive de bois pour la fabrication de charpentes et de meubles mais aussi pour le bois de chauffe et pour la technique de pêche "acadja". Dans la Commune d'Adjohoun, il a été montré que la régression des forêts est liée à l'utilisation des ressources forestières dans la fabrication du charbon de bois. Ainsi, Sinsin et Tenté (2002) affirment que l'homme, par ses diverses activités est le premier facteur de dégradation des formations végétales. Cette pression humaine a eu pour conséquence la diminution de la superficie forestière dans diverses régions du Bénin. En effet, selon Leroux (2012), les forêts ont perdu près de $24 \%$ de leur superficie initiale, avec une tendance qui s'est

\section{CONCLUSION}

Au terme de cette étude, l'analyse diachronique des cartes d'occupation du sol après une classification supervisée des images Landsat 1986, 2000 et 2013 a montré que la forêt communautaire d'Ahouanhouzoukan a été marquée par une perte importante de superficie des forêts riveraines à $C$.

\section{REFERENCES BIBLIOGRAPHIQUES}

Adjakpa BJ, 2006. Flore et végétation du Bas delta de I'Ouémé des zones humides du Sud-Bénin. Thèse de Doctorat de $3^{\text {ème }}$ Cycle de Biologie Végétale, Faculté des Sciences et Techniques, Université Cheikh Anta Diop, Dakar, Sénégal, 76 p. accélérée entre 1986 et 2000 dans la Commune de Djougou et entre 1973 à 2012 dans le bassin supérieur de l'Ouémé avec une perte de $47 \%$. De même, entre 1988 et 2008, Adjakpa (2012) a montré que 48,62 \% des formations végétales du Bas delta de l'Ouémé se sont dégradées. En outre, contrairement aux résultats de la présente étude qui montrent que les forêts riveraines ont évolué en forêts riveraines dégradées et formations marécageuses entre 1986 et 2013, Adjakpa (2012) a constaté plutôt que les forêts riveraines ont évolué en savane arbustive et savane herbeuse. En revanche, les forêts riveraines dégradées ont connu une augmentation spectaculaire de leur superficie entre 1986 et 2013 avec un taux de 33,40\% correspondant à 198,28 ha. Ce constat peut également s'expliquer par les principales activités anthropiques menées dans la forêt (abattage des arbres, pêche, recherche de bois de chauffe).

laurifolia et des jachères au profit des forêts riveraines à $M$. inermis et des forêts marécageuses. Cette perte de superficie est due aux activités anthropiques menées dans la forêt telles que l'abattage des arbres, la pêche, la recherche du bois de feu, l'écorçage des arbres, la cueillette des feuilles, les bois d'œuvre.

Adjakpa BJ, 2012. Habitat et avifaune des zones humides du Bas delta de l'Ouémé (SudBénin). Thèse de Doctorat d'Etat ès Sciences Naturelles, Faculté des Sciences et Techniques, Université Cheikh Anta Diop, Dakar, Sénégal, 134 p. 
Arouna O, Toko I, Djogbénou CP, Sinsin B, 2011. Comparative analysis of local populations perceptions of socio-economic determinants of vegetation degradation in soudano-guinean area in Benin (West Africa). International Journal of Biodiversity and Conservation 3 (7) : 327-337.

Aubreville A, 1957. Accord à Yangambi sur la nomenclature des types africains de végétation. Bois et forêts des tropiques 51 : 23-27.

Bogaert J, Barima YSS, Ji J, Jiang H, Bamba I, lyongo WML, Mama A, Nyssen E, Dahdouh-Guebas F, Koedam N, 2011. A methodological framework to quantify anthropogenic effects on landscape pattern. Landscape ecology in Asian cultures $n^{\circ} 45$, Springer, Verlag, New York, 141-167.

de Wasseige C, Flynn J, Louppe D, Hiol Hiol F, Mayaux P, 2013. Les forêts du bassin du Congo - Etat des forêts. $328 p$.

FAO, 2016. Situation des forêts du monde 2016. Forêts et agriculture: défis et possibilités concernant l'utilisation des terres. Rome. 138 p.

Geist HJ et Lambin EF, 2001. Land-Use and LandCover Change (LUCC) Project IV. International Human Dimensions Programme on Global Environmental Change (IHDP) V. International Geosphere-Biosphere Programme (IGBP), LUCC Report Series. 345-477.

Leroux T, 2012. Analyse diachronique de la dynamique paysagère sur le bassin supérieur de l'Ouémé (Bénin) à partir de l'imagerie Landsat et MODIS : Cas d'étude de la forêt communale de Djougou. Rapport, $47 \mathrm{p}$.

Noumi NV, Zapfack L, Sonke B, 2011. Ecological behaviour and biogeography of endemic species of the genus Piper L. in Africa : A case of the Guineo-Congolean region. African Journal of Plant Science 5 (4) : 248-263.

Orthmann B, 2005. Vegetation ecology of a woodlandsavanna mosaic in central Benin (West Africa) : Ecosystem analysis with a focus on the impact of selective logging. PhD Thesis, Rostock University, Allemagne. $137 \mathrm{p}$.

PDC, 2014. Plan de développement communal 2008 2014, rapport principal, $321 \mathrm{p}$.

Sinsin B et Tenté B, 2002. Diversité et structure des formations arborescentes du secteur PermaToucountouna dans la chaîne de l'Atacora (Bénin). Etude floristique de la végétation (8) : 31-42.
Tenté BA, 2005. Recherche sur les facteurs de la diversité floristique des versants du massif de I'Atacora : secteur Perma- Toucountouna (Bénin). Thèse de Doctorat, Université d'Abomey-Calavi, $252 \mathrm{p}$. 